\title{
Article
}

\section{Post discharge medicines use review service for older patients: recruitment issues in a feasibility study}

Rutter, Paul, Ramsbottom, Helen Frances and Fitzpatrick, Ray

Available at http://clok.uclan.ac.uk/14218/

Rutter, Paul ORCID: 0000-0003-4106-1515, Ramsbottom, Helen Frances and Fitzpatrick, Ray (2016) Post discharge medicines use review service for older patients: recruitment issues in a feasibility study. International Journal of Clinical Pharmacy, 38 (2). pp. 208-212. ISSN 2210-7703

It is advisable to refer to the publisher's version if you intend to cite from the work. http://dx.doi.org/10.1007/s11096-015-0243-8

For more information about UCLan's research in this area go to http://www.uclan.ac.uk/researchgroups/ and search for <name of research Group>.

For information about Research generally at UCLan please go to http://www.uclan.ac.uk/research/

All outputs in CLoK are protected by Intellectual Property Rights law, including Copyright law. Copyright, IPR and Moral Rights for the works on this site are retained by the individual authors and/or other copyright owners. Terms and conditions for use of this material are defined in the policies page. 


\section{International Journal of Clinical Pharmacy \\ Post Discharge Medicines Use Review (dMUR) Service for Older Patients: Recruitment Issues in a Feasibility Study \\ --Manuscript Draft--}

\begin{tabular}{|l|}
\hline Manuscript Number: \\
\hline Full Title: \\
\hline Article Type: \\
\hline Keywords: \\
\hline Corresponding Author: \\
\hline $\begin{array}{l}\text { Corresponding Author Secondary } \\
\text { Information: }\end{array}$ \\
\hline Corresponding Author's Institution: \\
\hline $\begin{array}{l}\text { Corresponding Author's Secondary } \\
\text { Institution: }\end{array}$ \\
\hline
\end{tabular}

First Author:

First Author Secondary Information:

Order of Authors:

Helen Frances Ramsbottom, MPharm

Ray Fitzpatrick, BSc (Pharm), PhD

Paul Rutter, BPharm, PhD

IJCP-D-15-00507R2 Issues in a Feasibility Study

\section{Short Research Report}

Helen Frances Ramsbottom, MPharm

Southport, UNITED KINGDOM

Helen Frances Ramsbottom, MPharm
Post Discharge Medicines Use Review (dMUR) Service for Older Patients: Recruitment

hospital discharge; older people; Recruitment; pharmacist; Medication Review

Southport and Ormskirk Hospitals NHS Trust

Southport and Ormskirk Hospitals NHS Trust

Order of Authors Secondary Information:

Funding Information:

Abstract:

\section{Abstract}

Background

The community pharmacy Medicines Use Review (MUR)service in England has been identified as a way of providing support with medication to recently discharged patients; however initial uptake of post-discharge Medicines Use Reviews has been low.

Objective

To identify barriers to recruitment into a randomised controlled feasibility study of a hospital referral system to older patients' regular community pharmacists.

Method

Ward pharmacists at Southport District General Hospital identified patients aged over 65 to be approached by a researcher to assess eligibility and discuss involvement in the trial. Participants were randomised to referral for a post discharge MUR with their regular community pharmacist, or to standard discharge care. Reasons for patients not participating were collected.

Results

Over a 9-month period 337 potential participants were identified by ward pharmacists. Of these, 132 were eligible and 60 were recruited.

Barriers to recruitment included competing priorities among ward pharmacists, and national restrictions placed on MURs e.g. housebound patients and those requiring carer support with medication. Lack of expected benefit resulted in a high proportion of patient refusals.

Conclusions

The current provisions for post discharge MURs exclude many older people from participation, including those possibly in greatest need.

Unfamiliarity with the role of the pharmacist in transitional care may have affected patients' perceived 'cost-benefit' of taking part in this study. 
Impact of Findings on Clinical Pharmacy Practice

*Engaging older patients in the hospital setting with the concept of a post discharge

Medicines Use Review in community pharmacy is challenging.

*Further support for hospital teams to identify and refer suitable patients for post discharge Medicines Use Reviews is necessary.

*The current provisions for post discharge medicines use reviews in England appear to cater poorly for patients who are housebound and those who rely on others to support them with their medicines at home.

\section{Response to Reviewers:}

Reviewer 1: Comments and Responses

1.In the abstract and on various other occasions throughout the manuscript, the participants are referred to as the 'elderly'. I would advise that this terminology be replaced with 'older adults' or 'older people', which would be regarded as more appropriate terms to use when referring to this population. Done

2.In Figure 1, please change '10682 Medical Patients' to '10682 medical patients' and '337 Patients' to '337 patients', (lowercase), in keeping with the word formatting in the other boxes within the same figure. Done

3.Line 68: 'Feedback from ward pharmacists via a focus group...' I would advise that you add $(n=x)$ or similar, to indicate how many ward pharmacists provided feedback in the focus group. Done $(n=7)$

4. Whilst this article predominantly focusses on recruitment being problematic due to it being a study attempting to recruit older adults, an important finding described in this research report was that, overall, the pharmacists only referred $3.2 \%$ of all patients admitted, which is a significantly lower proportion than one would expect given the wider patient population admitted to hospitals. As such, this clearly represented a major barrier to the recruitment process. As the author(s) states that recruitment of participants to the study was 'often forgotten about' by ward pharmacists (line 70), it is perhaps unclear to the reader what steps (if any) were taken to ensure that pharmacists were aware of their role in the recruitment process. This point is also pertinent as the author(s) also report that of the 337 patients referred to the researcher by the pharmacists, 105 were ineligible for inclusion as they did not meet the specified study inclusion criteria. As this represents nearly one third of all those who were referred, this would lead this reviewer to assume that the ward pharmacists involved in recruitment were not aware of the study's inclusion/exclusion criteria (as suggested in lines 35-36). To address this issue, I think this article would benefit from a comment in the discussion section to clarify exactly how and to what extent the pharmacists involved in the recruitment were informed of the recruitment process.

The following comment has now been inserted into the discussion: The low referral rate was despite steps taken by the researcher to raise awareness of the study among ward pharmacists, including a 'briefing' session prior to its commencement and posters displayed around the pharmacy department detailing types of patients who might be suitable. A study 'pack' was also given to each pharmacist, containing background information about the study and its eligibility criteria. Additionally, email reminders were sent regularly to all ward pharmacists throughout the recruitment period In addition, this article would be improved if the author(s) could provide a short comment on whether they believe the approach taken with involving pharmacists in the recruitment process could have been improved, and if so, how?

The following comment has now been inserted into the discussion: Further investigation of these barriers and possible solutions (for example, increased pharmacist time on wards to allow for patient screening or use of information technology to make referral easier) is ongoing.

\section{On line 98, please change 'pharmacist led' to 'pharmacist-led'. Done}

6.On lines 81-83, the author(s) argue that patients who have carers managing their medicines at home are 'frailer and more susceptible to adverse drug events'; this report would benefit from the inclusion of a reference here for this assertion, particularly as the author(s) also use this argument again to form part of their conclusion (line 128). If no specific reference exists to support the association, I would suggest that this sentence is re-worded to reflect that the proposed association is a (valid) assumption. No specific reference exists; first two paragraphs under subheading 'Patient exclusions' (lines 75-83 in original manuscript) have now been 
combined into a single paragraph to summarise the issue addressed (lines 91-96 in revised manuscript)

7.On lines $116-117$, please add (1.5\%) after 'only 2 patients...' as previous findings are presented in this way in the text (i.e. number followed by percentage in brackets).

Similarly, on line 118: '11 patients...', please add the percentage for information. Done

8.On lines 75-78 the author(s) state that polypharmacy is common among older adults who have 'managed care at home'. The relevance of this statement, in the context of patients' ineligibility, is not clear to this reviewer. I would ask the author(s) to review and either remove or rephrase this point in order to clarify their intended meaning. The intended argument here is: the fact that patients who had carers managing their medicines had to be excluded is a cause for concern and needs addressing in terms of future service development. The combination of first two paragraphs under subheading 'Patient exclusions' (lines 75-83 in original manuscript) attempts to clarify this point: In fact, patients requiring carers to manage their medication may be more in need of a medication support service, as polypharmacy, including the use of inappropriate medication, is common among older populations who have 'managed care at home' [8].

9.In Table 1 please correct 'Participants community pharmacy...' to 'Participant's community pharmacy...' Done

10.Finally, I would draw the author(s) attention to the reference section of the research report. Please review and correct the formatting of references as per the requirements of the journal. The use of semi-colons, commas, spaces, full-stops, capital letters and page numbering varies throughout the reference list. Also, some journals are abbreviated and others are not. Also, in reference number 8 , there is a ' 1 ' after 'Fialova D'. Reference list re-visited and re-formatted

Reviewer 2: Comments and Responses

Abstract:

1. In the methods section, please briefly state potential patients were visited by a researcher to assess eligibility and discuss involvement in the trial, and reasons for patients not participating were collected, or similar. Done

2. In the results section, please state that of the 337 potential participants, 132 were eligible and 60 were recruited. Done

3. Suggest first two sentences of conclusion are amended to summarise most common reasons and placed in the results section of the abstract. Done: following sentences now inserted in results: Barriers to recruitment included competing priorities among ward pharmacists, and national restrictions placed on Medicines Use Reviews, for example housebound patients and those requiring carer support with medication. Lack of expected benefit resulted in a high proportion of patient refusals.

Introduction:

4. If word count allows, it may assist international readers if the description of the MUR program was expanded. From memory the program is free for consumers - if so, this suggest this is stated. Lines 9-13 now read: In the UK, a national service known as Medicines Use Reviews (MUR) is offered by many community pharmacists which is free to patients and paid for centrally by the NHS. The MUR is a face-to-face consultation between a patient and pharmacist, designed to identify any problems or information needs patients have with their medicines and offer solutions [4].

5. Final sentence of introduction seems out of place (line 25) - suggest deleting or moving. Deleted, (along with preceding sentence in order to reduce word count)

6. The section of the aim which states "to investigate the logistics of setting up the study" could be a little more specific. Aim now reads: To identify barriers to recruitment into a randomised controlled feasibility study of a hospital referral system to older patients' regular community pharmacists, and identify areas for improvement of the service or study design.

Methods: 


\section{Line 43 (Exclusion criteria) suggest restarting numbering at 1. Done}

8. Can the authors please describe the process after eligibility was assessed ie did the researcher talk to the patients, what information was provided (ie brochure) etc The following text has been inserted (lines $44-47$ of revised manuscript): The researcher approached eligible patients to discuss involvement in the study and provide an information leaflet. Patients were given up to 24 hours (or longer if requested) to consider participation. Reasons for refusal were collected via verbal discussion, entered into an Excel spreadsheet and categorised by the researcher.

9. Please describe the method used to collect reasons for refusal ie simple discussion with patient or was a survey completed etc.

See response to point 8 above

Editor comments and Responses

- The results section in the abstract is not in line with the described Objective of the study.

Objective now reads: To identify barriers to recruitment into a randomised controlled feasibility study of a hospital referral system to older patients' regular community pharmacists. Results section also amended as per reviewer 2, comment 3.

- Please insert 3-5 Statements on the impact of your findings on practice, patients or professionals, and add them at the end of the abstract in Editorial Manager. Done

- The references to the reports need more detail, see the Vancouver style formatting instructions.

All references checked and now in the authors' opinion are correct - Replace the titles of some journals by the appropriate abbreviation (ref 9 and 10). Done 
Miss Helen Ramsbottom

Pharmacy Department, Southport and Ormskirk NHS Trust

Town Lane

Southport

PR8 6PN

School of Pharmacy

University of Wolverhampton

Wulfruna St

Wolverhampton

WV1 1SB

$14^{\text {th }}$ December 2015

Dear Editor,

Please accept this revised submission of a short research report:

Post Discharge Medicines Use Review (dMUR) Service for Older Patients: Recruitment Issues in a Feasibility Study.

The paper reports on the recruitment phase of a randomised controlled feasibility study of a postdischarge medicines support service for patients aged 65 and older. The study took place in England and involved referral of eligible patients from hospital to community pharmacy for post discharge Medicines Use Review (dMUR), a nationally commissioned service which has had low take-up in practice. Participants were randomised to referral for a dMUR, or to standard discharge care.

The authors feel this article sits well within the scope of the International Journal of Clinical Pharmacy as it describes the challenges faced by pharmacy practice researchers attempting to set up a controlled study of a medicines management service for one of the patient groups most vulnerable to adverse medication events; ie elderly patients who are being transferred from one care setting to another. This research is therefore of national importance within England but also of national importance in a world with an aging population who are taking more and more medicines.

\section{Submission Statement}

This article is not in submission elsewhere in any form. All authors have contributed significantly to the publication and are in agreement with this submission.

The authors are:

Miss Helen Ramsbottom, Clinical Pharmacist, Southport and Ormskirk Hospital NHS Trust and PhD researcher, University of Wolverhampton (Addresses as above)

Prof Ray Fitzpatrick, Professor of Clinical Pharmacy, School of Pharmacy, Keele University, Staffordshire, UK

Prof Paul Rutter, Professor of Pharmacy Practice, School of Pharmacy, Wolverhampton, UK

Main article length: 1530 words

Abstract length: 215 words

Number of references: 10; word count: 307

Yours sincerely

Helen Ramsbottom 
1 Word count 1530

2 Post Discharge Medicines Use Review (dMUR) Service for Older Patients: Recruitment

3

4

5

6

7

8

9

\section{Issues in a Feasibility Study}

\section{Introduction}

The likelihood that an older medical patient will be discharged from hospital on the same medication they were admitted on is less than $10 \%$, and this extensive medication regimen modification may predispose to an increased risk of mortality [1].

Improved discharge information and patient education are core principles for reducing unintentional medication changes, patient harm and hospital re-admissions [2,3]. In the UK, a national service known as Medicines Use Reviews (MUR) is offered by many community pharmacists which is free to patients and paid for centrally by the NHS. The MUR is a face-to-face consultation between a patient and pharmacist, designed to identify any problems or information needs patients have with their medicines and offer solutions [4].

Since 2011, the MUR service has targeted specific patient groups, identified as being at particular risk of medication related problems or poor adherence, including patients recently discharged from hospital [4]. However, early evidence suggests uptake of post discharge MURs (dMURs) has been poor; pilot studies have shown that less than $3 \%$ of patients 'signposted' to the service by hospital pharmacy staff receive a dMUR [5].

In 2012, a Department of Health steering group recommended that formal communication channels between hospital and community pharmacy should be established so that dMURs become an integral part of the medicines pathway [2]. However, at present, most NHS hospital Trusts do not appear to have such formalised systems in place [6].

\section{Aim}


24 To identify barriers to recruitment into a randomised controlled feasibility study of a hospital referral

25 system to older patients' regular community pharmacists, and identify areas for improvement of the 26 service or study design.

\section{Ethical Approval}

28 Ethics approval for the study was obtained from the Northwest Research Ethics Committee (Ref 29 13/NW/0779).

\section{Method}

31 All pharmacists working on medical wards at Southport and Ormskirk NHS Trust, England were asked to identify patients over the age of 65 years who in their professional opinion might benefit from receiving a dMUR. These patients were referred on to the researcher (HR), who visited each patient on the wards to assess eligibility. Inclusion criteria (All must be met for eligibility)

1. Taking at least one long-term medication prior to admission

2. Taking five or more medicines OR one or more changes to medication during hospital stay

3. Patient's community pharmacy signed up to received referrals from the hospital pharmacy.

\section{Exclusion Criteria}

1. Patient will have a relative or carer managing their medication once back at home

2. Patient unable to name a regular community pharmacy

3. Patient unable to visit their community pharmacy for an MUR (unless community pharmacy offers telephone or domiciliary MURs).

44 The researcher approached eligible patients to discuss involvement in the study and provide an information leaflet. Patients were given up to 24 hours (or longer if requested) to consider 
participation. Reasons for refusal were collected via verbal discussion, entered into an Excel spreadsheet and categorised by the researcher.

Consenting patients were randomised to receive a dMUR at their regular community pharmacy or standard hospital discharge care (no formal communication with the patient's community pharmacy, unless the patient received their medication in a weekly compliance aid (monitored dosage system) in which case a discharge list of medication was faxed to the community pharmacy, usually within 24 hours of discharge).

Prior to discharge each patient was told which group they had been assigned to, so they knew whether or not to expect their community pharmacist to contact them to arrange a dMUR.

\section{Results}

The study took place between $7^{\text {th }}$ April 2014 and $6^{\text {th }}$ January 2015. Over this 9-month period 337 potential participants were identified by ward pharmacists, which ultimately resulted in 60 patients recruited to the study, as shown in Figure 1.

\section{'Insert Figure 1 here'}

The reasons for exclusion and refusal are displayed in Table 1.

'Insert Table 1 here'

\section{Discussion}

This study is the first to describe formalising secondary care discharge of older patients into a nationally contracted community pharmacy service (dMURs) as advocated by UK health officials and bodies representing pharmacy.

Over the study period over 10,000 patients over 65 years of age were admitted to the hospital;

67 clearly a very small proportion (3.2\%) of these patients were identified by ward pharmacists and referred on to the researcher. This highlights the pivotal role ward staff play in such studies as 
identified by McMurdo et al in their review of recruitment of older people to research [7]. The low referral rate was despite steps taken by the researcher to raise awareness of the study among ward pharmacists, including a 'briefing' session prior to its commencement and posters displayed around the pharmacy department detailing types of patients who might be suitable. A study 'pack' was also given to each pharmacist, containing background information about the study and its eligibility criteria. Additionally, email reminders were sent regularly to all ward pharmacists throughout the recruitment period. Feedback from ward pharmacists $(n=7)$ via a focus group highlighted that identification of patients for inclusion into any type of trial was not standard practice, thus not part of their daily routine and was therefore often forgotten about. Other barriers identified by the pharmacists were time constraints and concerns over patients' poor health status. Further investigation of these barriers and possible solutions (for example, increased pharmacist time on wards to allow for patient screening or use of information technology to make referral easier) is ongoing.

\section{Patient Exclusions}

The most frequent reason (30.8\%) for ineligibility was patients' not self-medicating at home. This has implications for the English service specification for dMURs, which stipulates they must be conducted with the patient and not a care giver, unless the patient specifically consents for the carer to be present. In fact, patients requiring carers to manage their medication may be more in need of a medication support service, as polypharmacy, including the use of inappropriate medication, is common among older populations who have 'managed care at home' [8].

The nature of the nationally agreed service specification for community pharmacists also accounted for a relatively high proportion of patients ( $n=22,24.2 \%$ of exclusions) being excluded from the study due to being housebound and unable to attend a community pharmacy for a dMUR. Only around one in 10 community pharmacies in this study were able to provide domiciliary visits. Mobility issues are obviously a key consideration in this population; reduced mobility due to ill health or 
availability of transport has been cited by others as a challenge to the recruitment of older patients

95

96

97

98

101

102

103

104

105 $[7,9]$.

Twenty patients (22\% of exclusions), were not well enough to engage in discussion with the researcher at the time of contact, either because they were too unwell $(n=9)$ or had cognitive impairment $(n=11)$. Delirium or other conditions may temporarily impair older patients' capacity, but they may recover by the point of discharge [7]. Therefore some patients may require more than one visit to properly engage with them regarding research.

\section{Patient Refusals}

Of those patients fully screened, $72(32 \%)$ refused. This is within the range $(8-54 \%)$ found by McMurdo, and similar to a study of a pharmacist-led medication review clinic for older patients in primary care $(36 \%)[7,10]$.

The key reason for non-participation was patients saw no benefit in having a dMUR with their local community pharmacist $(n=20,27.8 \%)$. A further six patients (8.3\%) stated specifically that they would rather see their GP to discuss the changes to their medication. This indicates that the concept of the dMUR was either not fully understood by these patients, or not viewed as a useful service for them.

Fourteen (19.4\%) patients did not want to be involved in 'research'. Distrust of researchers and a reluctance to change their normal routine in order to take part in research have been cited elsewhere as a challenge in the recruitment of frail older patients [10].

Eleven patients (15.3\%) expressed concern over time commitment that the study imposed. Beyond the dMUR itself, the study included two subsequent follow-up postal questionnaires, up to 6 months after the initial discharge. Patients may have viewed this commitment too burdensome, although comments from these patients (such as 'l've got enough appointments') also suggested that even the initial referral for a dMUR was too onerous. Again, an unfamiliarity with the role of the 

part in this study.

However, it appears that old age, per se, may not have been a barrier to participation (only 2 patients (1.5\%) said this was the reason for not taking part), but rather the frailty and co-morbidity that may accompany it (11 patients (15.3\%) declined due to 'health reasons'). This is consistent with

123 research done by others $[7,10]$. This potentially biases the study sample toward those patients who 124 are more 'well' and thus may be less in need of a medication review.

\section{Conclusion}

127 This study has demonstrated that recruitment of older people to a study of a post discharge medicines support service provided by community pharmacists is particularly difficult for a number of reasons. Patient unwillingness to participate, either due to ill-health or perceived lack of benefit were noted. Additionally, pertinent to this study, the current provisions in England for post discharge MURs excluded many people from participation, including those who were possibly in greatest need. 


\section{References}

1. Mansur N, Weiss A, Beloosesky Y. Relationship of in-hospital medication modifications of elderly patients to postdischarge medications, adherence, and mortality. Ann Pharmacother. 2008 June 01;42(6):783-789.

2. Steering Group on Improving the Use of Medicines (for better outcomes and reduced waste). Improving the use of medicines for better outcomes and reduced waste: An action plan. Dept. of Health; 2012. https://www.gov.uk/government/uploads/system/uploads/attachment data/file/212837/Improvin g-the-use-of-medicines-for-better-outcomes-and-reduced-waste-An-action-plan.pdf. Accessed 14 December 2015.

3. Royal Pharmaceutical Society. Keeping patients safe when they transfer between care providers - getting the medicines right. Final Report. RPS; 2012. http://www.rpharms.com/currentcampaigns-pdfs/rps-transfer-of-care-final-report.pdf. Accessed 14 December 2015.

4. Pharmaceutical Services Negotiating Committee/NHS Employers. Medicines use review and prescription intervention service specification. PSNC and NHS Employers; 2013. http://psnc.org.uk/wp-content/uploads/2013/06/MUR-service-spec-Aug-2013changes FINAL.pdf. Accessed 14 December 2015.

5. Royal Pharmaceutical Society. Keeping patients safe when they transfer between care providers getting the medicines right. Individual reports from the Early Adopter Sites. RPS; 2012. http://www.rpharms.com/current-campaigns-pdfs/eas-finalreports.pdf. Accessed 14 December 2015.

6. Bhatti N, Blenkinsopp A, Devlin L, Farooq H, Kazi I, Mulla I et al. Community pharmacists' experiences of managing patients' medicines after discharge from hospital: a preliminary study of discharge medicines use reviews. Int J Pharm Prac. 2013;21(Supp2):86-87

7. McMurdo MET, Roberts H, Parker S, Wyatt N, May H, Goodman C, et al. Improving recruitment of older people to research through good practice. Age Ageing. 2011;40(6):659-665.

8. Fialová D, Topinková E, Gambassi G, Finne-Soveri H, Jonsson PV, Carpenter I et al. Potentially inappropriate medication use among elderly home care patients in Europe. JAMA. 2005;293(11):1348-1358

9. Baqir W, Desai N, Harker N, Vyas A, Copeland R, Burdon M, et al. Can targeted medicines use reviews support safe transfer of care from hospital to community? Int J Pharm Prac. 2012;20(Supp2):82-83

10. Petty DR, Zermansky AG, Raynor DK, Vail A, Lowe CJ, Freemantle $\mathrm{N}$ et al. "No thank you": why elderly patients declined to participate in a research study. Pharm World Sci. 2001;23:22-27 
Figure 1: Summary of Recruitment Phase of Study

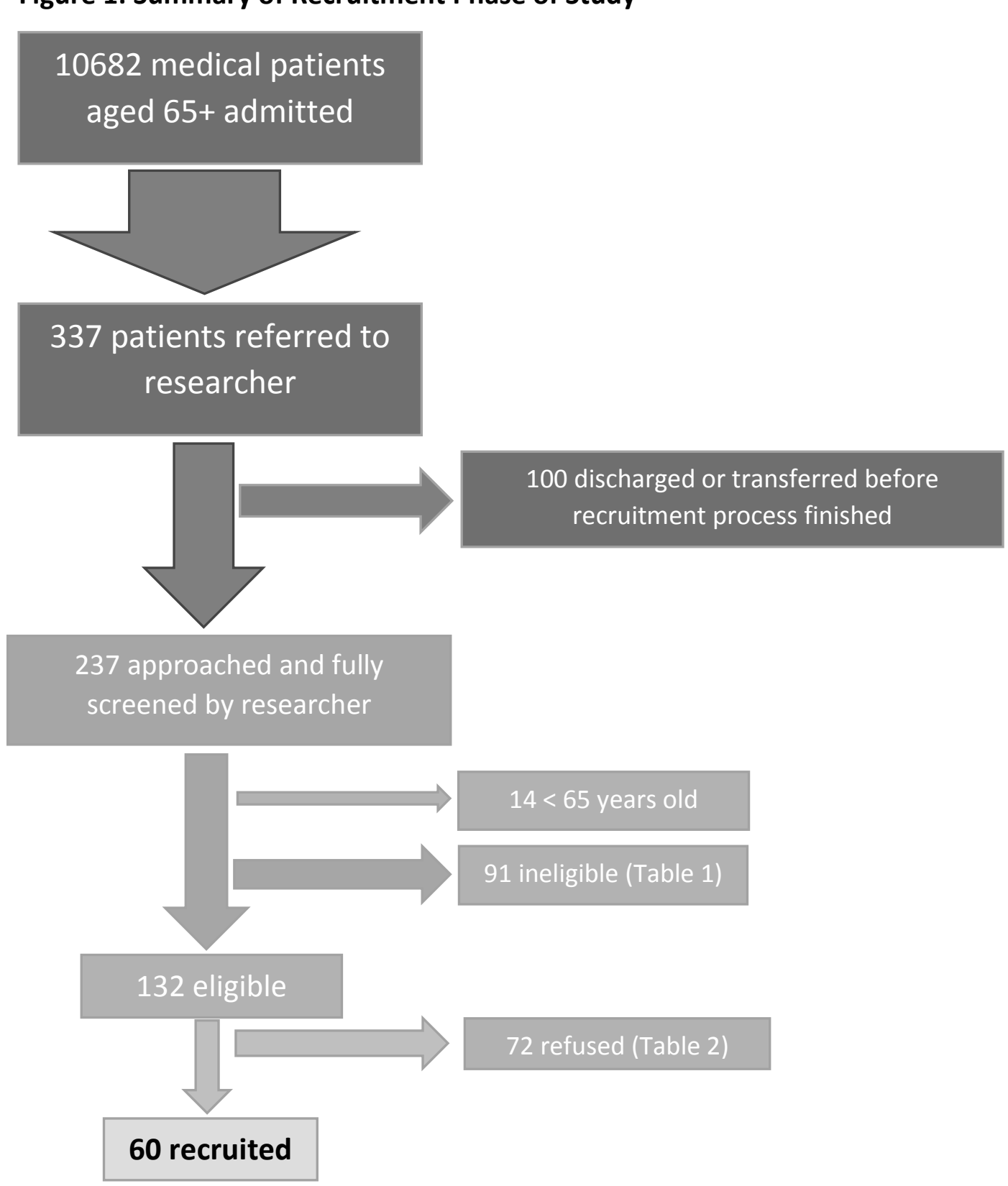

Click here to view linked References 
Table 1: Reasons for Ineligibility ( $n=91)$ and Refusal $(n=72)$

\begin{tabular}{|c|c|}
\hline Ineligibility & Number (\% of patients excluded) \\
\hline \multicolumn{2}{|l|}{ Protocol exclusions } \\
\hline Patient not self-medicating at home & $28(30.8 \%)$ \\
\hline Unable to access dMUR from chosen pharmacy & $22(24.2 \%)$ \\
\hline Being discharged out of study area & $11(12.1 \%)$ \\
\hline $\begin{array}{l}\text { Participant's community pharmacy not taking } \\
\text { part in the study }\end{array}$ & $2(2.2 \%)$ \\
\hline Patient does not use a regular pharmacy & $1(1.1 \%)$ \\
\hline \multicolumn{2}{|l|}{ Patient exclusions } \\
\hline Lack of capacity to consent & $11(12.1 \%)$ \\
\hline Too unwell to approach & $9(9.9 \%)$ \\
\hline MUR not needed & $7(7.7 \%)$ \\
\hline Reason for Refusal* & Number (\% of patients refusing) \\
\hline Patient sees no benefit to MUR & $20(27.8 \%)$ \\
\hline $\begin{array}{l}\text { Patient already has good links with community } \\
\text { pharmacy }\end{array}$ & $15(20.8 \%)$ \\
\hline Unwilling to engage with research & $14(19.4 \%)$ \\
\hline Health reasons & $11(15.3 \%)$ \\
\hline Time constraints & $11(15.3 \%)$ \\
\hline Would rather see GP & $6(4.5 \%)$ \\
\hline Hospital counselling adequate & $4(3 \%)$ \\
\hline $\begin{array}{l}\text { Patient does not want telephone MUR (home } \\
\text { visit not available and patient cannot attend } \\
\text { pharmacy) }\end{array}$ & $3(2.3 \%)$ \\
\hline $\begin{array}{l}\text { Patient does not agree with changes to } \\
\text { medicines }\end{array}$ & $2(1.5 \%)$ \\
\hline "Too old" & $2(1.5 \%)$ \\
\hline $\begin{array}{l}\text { Belief that medicines information will } \\
\text { automatically be transferred to community } \\
\text { pharmacy }\end{array}$ & $1(0.8 \%)$ \\
\hline
\end{tabular}


Peer review of manuscript entitled: Post Discharge Medicines Use Review (dMUR) Service for Older Patients: Recruitment Issues in a Feasibility Study

30/10/15

\section{Reviewer 1: Comments and Responses}

1. In the abstract and on various other occasions throughout the manuscript, the participants are referred to as the 'elderly'. I would advise that this terminology be replaced with 'older adults' or 'older people', which would be regarded as more appropriate terms to use when referring to this population. Done

2. In Figure 1, please change '10682 Medical Patients' to '10682 medical patients' and '337 Patients' to '337 patients', (lowercase), in keeping with the word formatting in the other boxes within the same figure. Done

3. Line 68: 'Feedback from ward pharmacists via a focus group...' I would advise that you add $(n=x)$ or similar, to indicate how many ward pharmacists provided feedback in the focus group. Done $(n=7)$

4. Whilst this article predominantly focusses on recruitment being problematic due to it being a study attempting to recruit older adults, an important finding described in this research report was that, overall, the pharmacists only referred $3.2 \%$ of all patients admitted, which is a significantly lower proportion than one would expect given the wider patient population admitted to hospitals. As such, this clearly represented a major barrier to the recruitment process. As the author(s) states that recruitment of participants to the study was 'often forgotten about' by ward pharmacists (line 70), it is perhaps unclear to the reader what steps (if any) were taken to ensure that pharmacists were aware of their role in the recruitment process. This point is also pertinent as the author(s) also report that of the 337 patients referred to the researcher by the pharmacists, 105 were ineligible for inclusion as they did not meet the specified study inclusion criteria. As this represents nearly one third of all those who were referred, this would lead this reviewer to assume that the ward 
Peer review of manuscript entitled: Post Discharge Medicines Use Review (dMUR) Service for Older Patients: Recruitment Issues in a Feasibility Study

30/10/15

pharmacists involved in recruitment were not aware of the study's inclusion/exclusion criteria (as suggested in lines $35-36)$. To address this issue, I think this article would benefit from a comment in the discussion section to clarify exactly how and to what extent the pharmacists involved in the recruitment were informed of the recruitment process.

The following comment has now been inserted into the discussion: The low referral rate was despite steps taken by the researcher to raise awareness of the study among ward pharmacists, including a 'briefing' session prior to its commencement and posters displayed around the pharmacy department detailing types of patients who might be suitable. A study 'pack' was also given to each pharmacist, containing background information about the study and its eligibility criteria. Additionally, email reminders were sent regularly to all ward pharmacists throughout the recruitment period In addition, this article would be improved if the author(s) could provide a short comment on whether they believe the approach taken with involving pharmacists in the recruitment process could have been improved, and if so, how?

The following comment has now been inserted into the discussion: Further investigation of these barriers and possible solutions (for example, increased pharmacist time on wards to allow for patient screening or use of information technology to make referral easier) is ongoing.

5. On line 98, please change 'pharmacist led' to 'pharmacist-led'. Done

6. On lines $81-83$, the author(s) argue that patients who have carers managing their medicines at home are 'frailer and more susceptible to adverse drug events'; this report would benefit from the inclusion of a reference here for this assertion, particularly as the author(s) also use this argument again to form part of their conclusion (line 128). If no specific reference exists to support the association, I would suggest that this sentence is re-worded to reflect that the proposed association is a (valid) assumption. No specific reference exists; first two paragraphs under subheading 'Patient 
Peer review of manuscript entitled: Post Discharge Medicines Use Review (dMUR) Service for Older Patients: Recruitment Issues in a Feasibility Study

30/10/15

exclusions' (lines 75-83 in original manuscript) have now been combined into a single paragraph to summarise the issue addressed (lines 91-96 in revised manuscript)

7. On lines $116-117$, please add (1.5\%) after 'only 2 patients...' as previous findings are presented in this way in the text (i.e. number followed by percentage in brackets). Similarly, on line 118: '11 patients...', please add the percentage for information. Done

8. On lines $75-78$ the author(s) state that polypharmacy is common among older adults who have 'managed care at home'. The relevance of this statement, in the context of patients' ineligibility, is not clear to this reviewer. I would ask the author(s) to review and either remove or rephrase this point in order to clarify their intended meaning. The intended argument here is: the fact that patients who had carers managing their medicines had to be excluded is a cause for concern and needs addressing in terms of future service development. The combination of first two paragraphs under subheading 'Patient exclusions' (lines 75-83 in original manuscript) attempts to clarify this point: In fact, patients requiring carers to manage their medication may be more in need of a medication support service, as polypharmacy, including the use of inappropriate medication, is common among older populations who have 'managed care at home' [8].

9. In Table 1 please correct 'Participants community pharmacy...' to 'Participant's community pharmacy...' Done

10. Finally, I would draw the author(s) attention to the reference section of the research report. Please review and correct the formatting of references as per the requirements of the journal. The use of semi-colons, commas, spaces, full-stops, capital letters and page numbering varies throughout 
Peer review of manuscript entitled: Post Discharge Medicines Use Review (dMUR) Service for Older Patients: Recruitment Issues in a Feasibility Study

30/10/15

the reference list. Also, some journals are abbreviated and others are not. Also, in reference number 8 , there is a ' 1 ' after 'Fialova $D$ '. Reference list re-visited and re-formatted

\title{
Reviewer 2: Comments and Responses
}

\author{
Abstract:
}

1. In the methods section, please briefly state potential patients were visited by a researcher to assess eligibility and discuss involvement in the trial, and reasons for patients not participating were collected, or similar. Done

2. In the results section, please state that of the 337 potential participants, 132 were eligible and 60 were recruited. Done

3. Suggest first two sentences of conclusion are amended to summarise most common reasons and placed in the results section of the abstract. Done: following sentences now inserted in results: Barriers to recruitment included competing priorities among ward pharmacists, and national restrictions placed on Medicines Use Reviews, for example housebound patients and those requiring carer support with medication. Lack of expected benefit resulted in a high proportion of patient refusals.

Introduction:

4. If word count allows, it may assist international readers if the description of the MUR program was expanded. From memory the program is free for consumers - if so, this suggest this is stated. Lines 9-13 now read: In the UK, a national service known as Medicines Use Reviews (MUR) is offered by many community pharmacists which is free to patients and paid for centrally by the NHS. The MUR is a face-to-face consultation between a patient and pharmacist, designed to identify any problems or information needs patients have with their medicines and offer solutions [4]. 
Peer review of manuscript entitled: Post Discharge Medicines Use Review (dMUR) Service for Older Patients: Recruitment Issues in a Feasibility Study

30/10/15

5. Final sentence of introduction seems out of place (line 25) - suggest deleting or moving. Deleted, (along with preceding sentence in order to reduce word count)

6. The section of the aim which states "to investigate the logistics of setting up the study" could be a little more specific. Aim now reads: To identify barriers to recruitment into a randomised controlled feasibility study of a hospital referral system to older patients' regular community pharmacists, and identify areas for improvement of the service or study design.

Methods:

7. Line 43 (Exclusion criteria) suggest restarting numbering at 1. Done

8. Can the authors please describe the process after eligibility was assessed ie did the researcher talk to the patients, what information was provided (ie brochure) etc

The following text has been inserted (lines 44-47 of revised manuscript): The researcher approached eligible patients to discuss involvement in the study and provide an information leaflet. Patients were given up to 24 hours (or longer if requested) to consider participation. Reasons for refusal were collected via verbal discussion, entered into an Excel spreadsheet and categorised by the researcher.

9. Please describe the method used to collect reasons for refusal ie simple discussion with patient or was a survey completed etc.

See response to point 8 above 
Peer review of manuscript entitled: Post Discharge Medicines Use Review (dMUR) Service for Older Patients: Recruitment Issues in a Feasibility Study

30/10/15

\section{Editor comments and Responses}

- The results section in the abstract is not in line with the described Objective of the study.

Objective now reads: To identify barriers to recruitment into a randomised controlled feasibility study of a hospital referral system to older patients' regular community pharmacists. Results section also amended as per reviewer 2 , comment 3.

- Please insert 3-5 Statements on the impact of your findings on practice, patients or professionals, and add them at the end of the abstract in Editorial Manager. Done

- The references to the reports need more detail, see the Vancouver style formatting instructions.

All references checked and now in the authors' opinion are correct

- Replace the titles of some journals by the appropriate abbreviation (ref 9 and 10). Done 